\title{
ON THE SYMBIOTIC X-RAY BINARY NATURE OF THE STAR CGCS 5926
}

\author{
N. Masetti ${ }^{1}$, U. Munari ${ }^{2}$, A. A. Henden ${ }^{3}$, K. L. Page ${ }^{4}$, J. P. Osborne ${ }^{4}$ \\ and S. Starrfield ${ }^{5}$ \\ 1 INAF - Istituto di Astrofisica Spaziale e Fisica Cosmica di Bologna, Via Gobetti \\ 101, I-40129, Bologna, Italy \\ 2 INAF - Osservatorio Astronomico di Padova, Sede di Asiago, \\ Via dell'Osservatorio 8, I-36012, Asiago, Italy \\ 3 AAVSO, 49 Bay State Road, Cambridge, MA 02138, U.S.A. \\ 4 Department of Physics and Astronomy, University of Leicester, Leicester, \\ LE1 7RH, United Kingdom \\ 5 School of Earth and Space Exploration, Arizona State University, Tempe, \\ AZ 85287-1404, U.S.A.
}

Received: 2011 August 2; accepted: 2011 September 1

\begin{abstract}
We report on multiwavelength (from X-ray to optical) follow up observations of a carbon star CGCS 5926, motivated by the fact that it is positionally coincident with a faint X-ray source of the ROSAT catalog, thus suggesting its possible symbiotic X-ray binary (SyXB) nature. Our optical spectroscopy confirms that this is a carbon star of type $\mathrm{C}(6,2)$. This allows us to infer for CGCS 5926 a distance of $\sim 5 \mathrm{kpc} . B V R_{\mathrm{C}} I_{\mathrm{C}}$ photometry of the star shows variability of $\sim 0.3 \mathrm{mag}$ with a periodicity of 151 days, which we interpret as due to radial pulsations. The source is not detected with the Swift satellite in X-rays down to a $0.3-10 \mathrm{keV}$ luminosity of $\sim 3 \times 10^{32} \mathrm{erg} \mathrm{s}^{-1}$. This nondetection is apparently in contrast with the ROSAT data; however, the present information does not rule out that CGCS 5926 can be a SyXB. This will be settled by more sensitive observations at high energies.
\end{abstract}

Key words: stars: carbon, AGB - stars: individual (CGCS 5926) - stars: oscillations

\section{INTRODUCTION: CGCS 5926}

Symbiotic X-ray binaries (SyXBs; e.g., see Masetti et al. 2006) are a small subclass of low-mass X-ray binaries in which (by analogy with the symbiotic binaries which are formed by an evolved late-type star and a white dwarf) the donor star is actually a red giant rather than a late-type dwarf.

Observationally, these systems are characterized by appreciable X-ray emission $\left(10^{32}-10^{34} \mathrm{erg} \mathrm{s}^{-1}\right.$; see Masetti et al. 2007 and references therein) positionally associated with a red giant star which spectroscopically does not show any peculiar features in the optical range. X-ray pulsations with periods ranging from hundreds up to tens of thousands of seconds are detected from these systems: this indicates that the accreting compact object is a slowly rotating neutron star. 


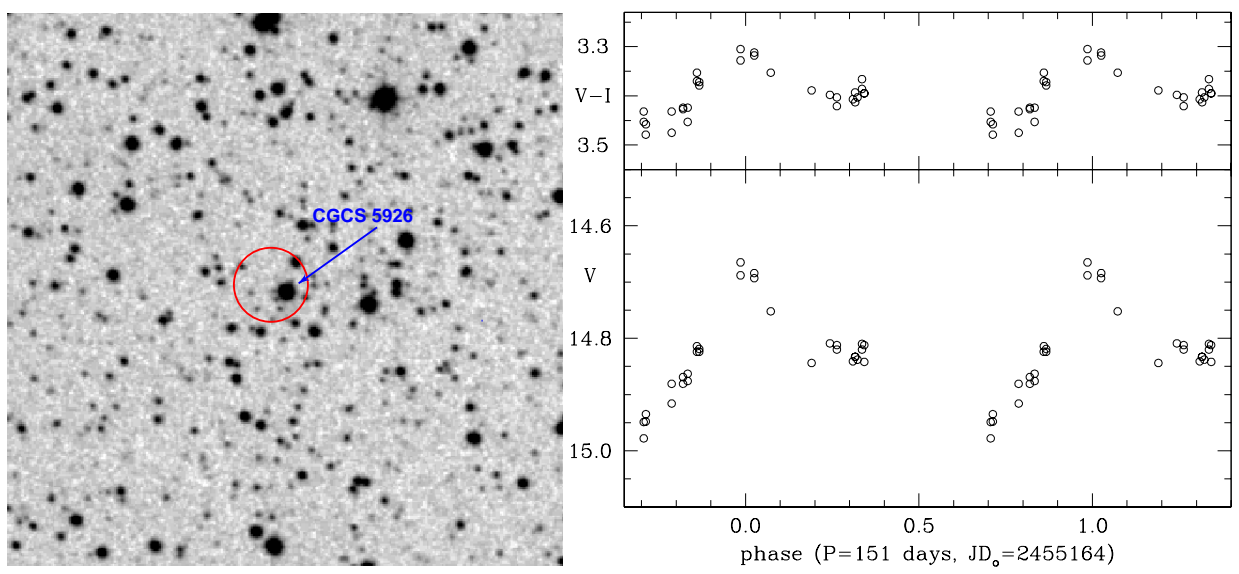

Fig. 1. Left panel. DSS-II-Red image of the field of CGCS 5926 (the star is indicated by the arrow) with superposed the $20^{\prime \prime}$ radius ROSAT X-ray error circle. North is at top and East is to the left; the field size is $5^{\prime} \times 5^{\prime}$. Right panel. $V$ and $V-I_{\mathrm{C}}$ light and color curves of CGCS 5926 folded with a period of 151 days and with zero phase at JD 2455164. We identify this as the pulsation periodicity of the star.

All the above characteristics make these systems rather unusual and, indeed, they are quite rare: up to now, only 6 systems are firmly included in this subclass of LMXBs (see Masetti et al. 2007; Nespoli et al. 2010 and references therein).

Here we focus our attention on the star CGCS 5926, which was classified by Maehara \& Soyano (1987) as a $V=14.8$ mag carbon star (thus a late-type giant) in the Cassiopeia region. Our interest in this star was drawn by the fact that (Figure 1, left) it is positionally inside the $20^{\prime \prime}$ error circle of the X-ray source 1RXS J234545.9+625256 belonging to the ROSAT All-Sky Survey Faint Source Catalog (Voges et al. 2000), which makes CGCS 5926 a SyXB candidate due to its optical spectral classification and its possible X-ray emission.

However, the lack of further information at optical, X-ray and other wavelengths does not make a secure case for inclusion in the SyXB subclass. It is therefore worthy of further analysis by means of a specific spectrophotometric campaign in the optical range, as well as of a pointed observation with the use of an X-ray satellite affording localizations with a precision better than a few arcseconds.

\section{OBSERVATIONS}

Optical spectroscopy of CGCS 5926 was acquired on 2009 October 14 with the $1.82 \mathrm{~m}$ "Copernicus" telescope of the Asiago Astronomical Observatory (Italy). The used setup provided a dispersion of $4.2 \AA /$ pixel. The total exposure time was $2 \times 20$ min centered at 02:03 UT.

The $B V R_{\mathrm{C}} I_{\mathrm{C}}$ imaging data of the source were obtained with the robotic 14-inch Celestron telescope of the Sonoita Research Observatory (New Mexico, U.S.A.) in 2009 from October 15 to December 27 and in 2010 from September 5 to November 17.

The field of CGCS 5926 was then observed on 2010 January 6 in the 0.3 $10 \mathrm{keV}$ band with the X-ray telescope (XRT; Burrows et al. 2005) on board 


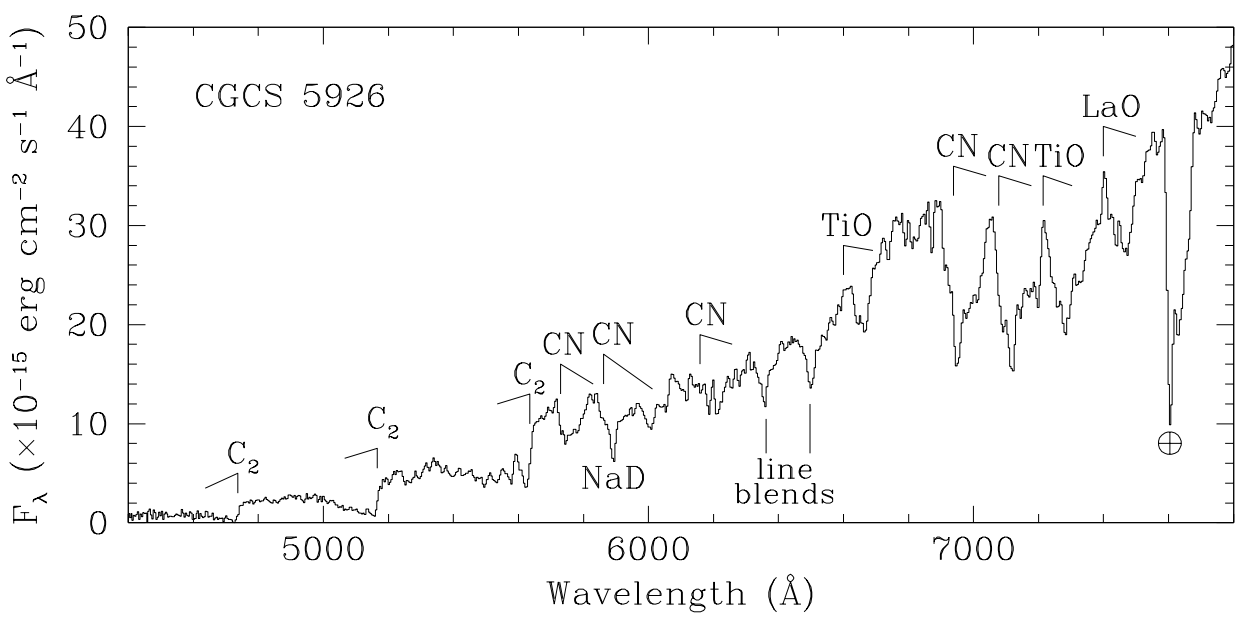

Fig. 2. A 4400-7800 $\AA$ optical spectrum of the star CGCS 5926 obtained with the 'Copernicus' telescope on 2009 October 14. The spectrum is typical of a late-type giant carbon star (see the text). The telluric absorption band at $7600 \AA$ is marked with the symbol $\oplus$.

of the Swift satellite (Gehrels et al. 2004). The pointing started at 08:34 UT, and $4374 \mathrm{~s}$ of on-source data were collected. In parallel with the X-ray pointing, the UltraViolet-Optical Telescope (UVOT; Roming et al. 2005) onboard Swift observed CGCS 5926 in the $U V M 2$ band $(\lambda=2246 \AA$; FWHM $=498 \AA)$ for a total of $4364 \mathrm{~s}$ starting at 08:37:42 UT of 2010 January 6.

\section{RESULTS}

The optical spectrum of CGCS 5926 (Figure 2) clearly shows the typical features of a carbon star (e.g., Yamashita 1967): it is dominated by the C2 Swan bands at 5165, 5635 and $6192 \AA$ in the blue, and by $\mathrm{CN}$ bands redwards. No emission features typical of X-ray binaries, such as Balmer and HeII lines, are present.

Using the two-dimensional $\mathrm{C}(m, n)$ diagnostics (Yamashita 1967; Cohen 1979) we can classify the spectral type of CGCS 5926 as C(6,2), which means an effective blackbody temperature $T_{\text {eff }} \sim 2500 \mathrm{~K}$ for this star according to Cohen (1979).

Our photometry indicates that the source shows a variability of amplitude $\Delta m \sim 0.3 \mathrm{mag}$ on timescales of tens of days and gets bluer with increasing brightness, as expected for radial pulsators. A periodicity search using the Fourier code of Deeming (1975) gives a single, strong probability peak at a period of 151 days (Figure 1, right, for the phased light curve). We interpret it as the radial pulsation period of the star. The ephemeris we obtained with this period, expressed in Heliocentric Julian Days (HJDs), provides the following times of maxima in the $V$ band:

$$
\mathrm{T}_{\max , V}=2455164( \pm 3)+(151 \pm 2) \times E,
$$

where $E$ is an integer number.

No X-ray source was detected in the XRT pointing: assuming a Crab-like 
spectrum, we get a $3 \sigma$ confidence observed flux limit of $\sim 9 \times 10^{-14} \mathrm{erg} \mathrm{cm}^{-2} \mathrm{~s}^{-1}$ in the $0.3-10 \mathrm{keV}$ energy range. Likewise, no UV source was detected in coincidence of the target down to a $3 \sigma$ limit of 21.8 mag in the $U V M 2$ band.

\section{CONCLUSIONS}

Using the available photometry, and given that a carbon star of spectral type $\mathrm{C}(6,2)$ has an absolute magnitude $M_{V} \approx-2.6$ (Cohen 1979), we derive a distance $d \approx 5.2 \mathrm{kpc}$ to CGCS 5926. This implies an upper limit to its X-ray luminosity of $\sim 3 \times 10^{32} \mathrm{erg} \mathrm{s}^{-1}$. This may cast doubts on the ROSAT detection and even on the possibility that this source can be a SyXB.

Actually, the lowest fluxes detected in X-rays from this type of source (Masetti et al. 2007; Nespoli et al. 2010), are of the order of the above limit for CGCS 5926. Thus the X-ray non-detection does not in itself allow us to rule out that this star is the optical counterpart of a SyXB. Indeed, given the size of carbon stars (e.g., Cohen 1979), the possibility of an eclipse of the X-ray accretor at the time of the Swift observation cannot be discarded.

Future spectrophotometric optical and near-infrared monitoring over a long time baseline (years) would help to determine if CGCS 5926 indeed belongs of a binary system, while deeper, longer and higher sensitivity X-ray observations would provide a possible detection of, or at least tighter upper limits on, the high-energy emission from this source.

ACKNOWLEDGMENTS. We thank A. Siviero, G. Cetrulo, A. Englaro and A. Milani for assistance in obtaining the optical spectrum of CGCS 5926, and the organizers of this meeting for their help in printing and displaying the poster. This research has made use of the ASI Science Data Center Multimission Archive; it also used the NASA-ADS Abstract Service. The use of the Simbad, Vizier and 2MASS databases is acknowledged. NM acknowledges financial contribution from the ASI-INAF agreement No. I/009/10/0. KLP and JPO acknowledge the support of the UK Space Agency. SS acknowledges partial support from NSF and NASA grants to ASU.

\section{REFERENCES}

Burrows D. N., Hill J. E., Nousek J. A. et al. 2005, Space Sci. Rev., 120, 165

Cohen M. 1979, MNRAS, 186, 837

Deeming T. J. 1975, Ap\&SS, 36, 137

Gehrels N., Chincarini G., Giommi P. et al. 2004, ApJ, 611, 1005

Maehara H., Soyano T. 1987, Ann. Tokyo Astron. Obs., 21, 293

Masetti N., Orlandini M., Palazzi E. et al. 2006, A\&A, 453, 295

Masetti N., Landi R., Pretorius M. L. et al. 2007, A\&A, 470, 331

Nespoli E., Fabregat J., Mennickent R. E. 2010, A\&A, 516, A94

Roming P. W. A., Kennedy T. E., Mason K. O. et al. 2005, Space Sci. Rev., 120, 95

Voges W., Aschenbach B., Boller T. et al. 2000, IAU Circ., 7432

Yamashita Y. 1967, Publ. DAO, 13, 67 